\title{
Folinsäure als Wuchsstoff bei verschiedenen Bakterien; ein neuer Folinsäuretest mit Staphylococcus aureus
}

\author{
Von Ernst-Friedrich Möller, Friedrich Weygand und Adolf Wacker
}

Aus dem Kaiser-Wilhelm-Institut für medizinische Forschung, Institut für Chemie, Heidelberg, und dem Chemischen Institut der Universität Heidelberg

(Z. Naturforschg. 4 b. 92-96 [1949]; eingegangen am 28. März 1949)

\begin{abstract}
Es' wurde das Folinsäurebedürfnis von Milchsäurebakterien, Staphylokokken, Streptokokken, Proteus-, Coli-, Pyocyaneus-, Fluorescens-, Dysenterie-, Typhus- und DiphtherieStämmen untersucht. Es ergab sich, daß nicht nur ein Teil der Milchsäurebakterien, sondern auch 40\% der geprüften Staphylokokkenstämme Folinsäure unbedingt zum Wachstum benötigen. Es konnte ein neuer Test zur Folinsäurebestimmung mit Staph. aureus ausgearbeitet werden, der dem bekannten Test mit Sc.faecalis $\mathrm{R}$ in Empfindlichkeit und Spezifität zumindest gleichwertig ist.
\end{abstract}

Z ur Ausweitung unserer früheren Versuche ${ }^{1}$ über dieHemmung des Wachstums von Streptococcus faecalis $\mathrm{R}$ durch 2-Amino-6-oxy-pteridinaldehyd-(8) und 2-Amino-6-oxy-8-d-arabo-tetraoxybutyl-pteridin und zur Prüfung der Frage, ob die Hemmung auch bei Folinsäure synthetisierenden Stämmen erfolgt, war es notwendig, eine Reihe von Bakterienstämmen auf ihre Folinsäurebedürftigkeit hin zu untersuchen.

\section{Methodik}

$\mathrm{Nährmedium} \mathrm{:} \mathrm{In} \mathrm{der} \mathrm{Absicht,} \mathrm{möglichst} \mathrm{viele}$ Bakterienstämme unter gleichen Bedingungen $\mathrm{zu}$ untersuchen, versuchten wir das in unserer vorigen Arbeit ${ }^{1}$ benutzte Nährmedium für $S c$. faecalis $\mathrm{R} \mathrm{zu}$ verwenden. Das Nährmedium enthielt alle von J. Te ply und C. A. E l v e h j e ${ }^{2}$ angegebenen Substanzen, mit Ausnahme des mit Norit behandelten Peptons. Als' Aminosäurequelle diente mit Pankreatin abgebautes Casein $^{3}$ (durch Behandeln mit Aktivkohle folinsäurefrei gemacht).

Das' Nährmedium mit bzw. ohne Folinsäure wurde $1 / 2$ Stde. bei $100^{\circ}$ sterilisiert. Stämme: Ihre Herkunft ist, wenn nichts anderes angegeben, bei Möller und Knoevenage ${ }^{4}$ verzeichnet. Die Stämme wurden im allgemeinen auf Agar gehalten, wenn nicht, wie bei einigen pathogenen Bakterien, Spezialnährmedien erforderlich waren, und etwa jeden Monat auf neuen Agar überimpft. Zwecks Beimpfung der Versuchsröhrchen wurden Vorpassagen angelegt, zuerst in Glucose-Pepton (Dif co + M e r ck), dann in synthetischem Medium + Folinsäure $\left(1,6 \mathrm{~m} \gamma / \mathrm{cm}^{3}=1,6 \cdot 10^{-9} \mathrm{~g} / \mathrm{cm}^{3}\right)$, in dem alle untersuchten Stämme gutes' Wachstum zeigten (mit Ausnahme von mehreren geprüften

1 F. We y g and, E. F. Mölle r u. A. Wa cker, Z. Naturforschg. 3 b, 299 [1948].

2 J. Te ply u. C. A. Elveh je m, J. biol. Chemistry 157, 303 [1945].

\begin{tabular}{|c|c|c|}
\hline Substanz & $\begin{array}{c}\text { Konz. pro } \\
\mathrm{cm}^{3}\end{array}$ & Bemerkungen \\
\hline $\mathrm{KH}_{2} \mathrm{PO}_{4}$ & $2,5 \mathrm{mg}$ & \\
\hline Na-Citrat & $25 \mathrm{mg}$ & \\
\hline Casein & $5 \mathrm{mg}^{*}$ & $\begin{array}{l}\text { mit Pankreatin } \\
\text { [verdaut }\end{array}$ \\
\hline Glucose & $20 \mathrm{mg}^{*}$ & Rohrzuckerhydro- \\
\hline Cystein-hydrochlorid & $0,2 \mathrm{mg}$ & {$\left[\right.$ nach $^{4}$} \\
\hline Adenin & $0,01 \mathrm{mg}$ & \\
\hline Guanin & $0,01 \mathrm{mg}$ & \\
\hline Uracil & $0,01 \mathrm{mg}$ & \\
\hline Xanthin & $0,01 \mathrm{mg}$ & - \\
\hline Asparagin & $0,1 \mathrm{mg}$ & \\
\hline$d, l$-Alanin & $0,2 \mathrm{mg}$ & \\
\hline Aneurin & $0,2 \gamma$ & \\
\hline Lactoflavin & $0,2 \gamma$ & \\
\hline Nicotinsäure & $0,6 \gamma$ & \\
\hline Adermin & $1,2 \gamma$ & \\
\hline Ca-Pantothenat & $0,4 \gamma$ & \\
\hline Biotin & $0,0004 \gamma^{*}$ & Methylester \\
\hline$p$-Aminobenzoesäure & $0,01 \gamma$ & [verseift \\
\hline $\mathrm{MgSO}_{4} \cdot 7 \mathrm{H}_{2} \mathrm{O}$ & $200 \gamma$ & \\
\hline $\mathrm{NaCl}$ & $100 \gamma$ & \\
\hline Fe-Citrat & $100 \gamma$ & \\
\hline Mn-Sulfat & $6,7 \gamma$ & \\
\hline
\end{tabular}

* Auf Ausgangsmaterial berechnet.

Tab. 1. Zusammensetzung des Nährmediums.

Gonokokkenstämmen). Die Versuchsröhrchen, die einmal keine, das andere Mal 1,6 $\mathrm{m} \gamma / \mathrm{cm}^{3}$ Folinsäure enthielten, wurden mit 2 Ösen (Durchm. $2 \mathrm{~mm}$ ) einer 24-stdg. Kultur aus der oben erwähnten zweiten Zwischenpassage beimpft. Die Stärke des Wachstums wurde durch Trübungsmessungen im Lange-Photometer gemessen. Bebrütungstemperaturen: s. Tabellen.

3 J. Teply u. C. A. Elveh jem; Analyt. Chemistry 19, 358 [1947]; E. C. Roberts u. E. E. Snell, J. biol. Chemistry 163, 499 [1946].

${ }_{4}^{4}$ E. F. M öll e r u. C. K n o e v e $\mathrm{n}$ a g e l, Klin. W., im Druck [1949]. 


\begin{tabular}{|c|c|c|c|c|}
\hline Bacterium & Stamm & $\begin{array}{l}\text { Bebrütungs- } \\
\text { temperatur }\end{array}$ & $\begin{array}{l}\text { Folinsäure- } \\
\text { bedürfnis }\end{array}$ & $\begin{array}{l}\text { Herkunft der } \\
\text { Stämme }\end{array}$ \\
\hline $\begin{array}{l}\text { Streptococcus faecalis } \mathrm{R} \\
\text { Streptococcus faecalis } \\
\text { Lactobacillus casei } \\
\text { Lactobacillus helveticus } \\
\text { Streptococcus thermophilus } \\
\text { Streptococcus thermophilus } \\
\text { Betacoccus arabinosus } \\
\text { Streptobacterium casei } \\
\text { Streptobacterium casei } \\
\text { Streptobacterium plantarum } \\
\text { Streptobacterium plantarum } \\
\text { Streptobacterium plantarum } \\
\text { Streptobacterium plantarum }\end{array}$ & $\begin{array}{l}\text { N.C.T.C.Nr.6459 } \\
\text { N.C.T.C.Nr.6357 } \\
\text { St.K 1 } \\
\text { St.K } 2 \\
\text { a } 11 \\
\text { C }_{6} \text { I } \\
\text { C }_{6} \text { III } \\
10 \mathrm{~S} \\
10 \mathrm{~S} \text { OJ } \\
\text { P } 24 \\
\text { P } 32\end{array}$ & 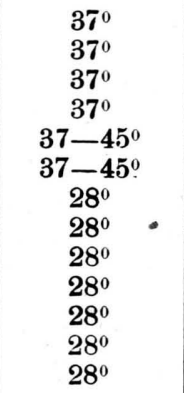 & $\begin{array}{l}+ \\
+ \\
+ \\
+ \\
+ \\
+ \\
\times \\
0 \\
0 \\
0 \\
0 \\
0 \\
0\end{array}$ & $\begin{array}{l}\text { U.S. Stamm } \\
\text { N.C.T.C. } \\
\text { U.S. Stamm } \\
\text { N.C.T.C. } \\
\text { \{isoliert aus Schweizer- } \\
\text { käse April 1947 } \\
\text { Prof.Orla Jensen, Kopenh. } \\
\text { Prof.Orla Jensen, Kopenh. } \\
\text { Prof.OrlaJensen, Kopenh. } \\
\text { Prof.Orla Jensen, Kopenh. } \\
\text { Prof.Orla.Jensen, Kopenh. }\end{array}$ \\
\hline
\end{tabular}

Tab. 2. Folinsäurebedürfnis von Milchsäurebakterien. Zeichener̀klärung: (+) benötigt Folinsäure; (X) Zuwachseffekt durch Folinsäure; (0) braucht keine Folinsäure.

\begin{tabular}{|c|c|c|c|}
\hline Bacterium & Stamm & $\begin{array}{l}\text { Folinsäure- } \\
\text { bedürfnis }\end{array}$ & Herkunft der Stämme ${ }^{4}$ \\
\hline $\begin{array}{l}\text { Staph. aureus haemolyticus } \\
\text { Staph. aureus } \\
\text { Staph. aureus haemolyticus } \\
\text { Staph. aureus } \\
\text { Staph. aureus } \\
\text { Staph. aureus } \\
\text { Staph. aureus } \\
\text { Staph. aureus } \\
\text { Staph. aureus } \\
\text { Staph. aureus } \\
\\
\text { Staph. aureus haemolyticus } \\
\text { Staph. aureus } \\
\text { Staph. aureus haemolyticus } \\
\text { Staph. aureus haemolyticus } \\
\text { Staph. aureus haemolyticus } \\
\text { Staph. aureus } \\
\text { Staph. aureus (Micrococcus?) } \\
\text { Staph. aureus +albus(haemolytisch) } \\
\text { Staph. albus } \\
\text { Staph. albus haemolyticus } \\
\text { Staph. albus } \\
\text { Staph. albus haemolyticus } \\
\text { Staph. albus } \\
\text { Staph. albus } \\
\text { Staph. albus }\end{array}$ & $\begin{array}{l}\text { KiAu (1517) } \\
\text { S } \\
1548 \mathrm{Au} \\
\mathrm{N}_{1} \mathrm{C} \\
1521 \mathrm{Au} \\
\mathrm{Bi} \mathrm{Au} \\
\mathrm{Knoll} \mathrm{Au} \\
\mathrm{WaAu} \\
\mathrm{K} 31 \mathrm{l} \\
\mathrm{Be} \\
\\
1520 \\
\mathrm{Bie} \\
\mathrm{Do} \\
\mathrm{Bo} \\
1648 \mathrm{Au} \\
\text { Stei } \\
\text { IG Au } \\
\mathrm{Bu} \mathrm{A} \mathrm{Au} \\
1646 \mathrm{~A} \\
1531 \mathrm{~A} \\
1833 \mathrm{~A} \\
1648 \mathrm{~A} \\
1837 \mathrm{~A} \\
\text { WA } \\
\mathrm{AI}\end{array}$ & $\begin{array}{l}+ \\
+ \\
\times \\
\times \\
\times \\
+ \\
\times \\
+ \\
\times \\
+ \\
\\
0 \\
\times \\
+ \\
0 \\
0 \\
0 \\
+ \\
0 \\
0 \\
0 \\
+ \\
+ \\
+ \\
\times \\
0\end{array}$ & 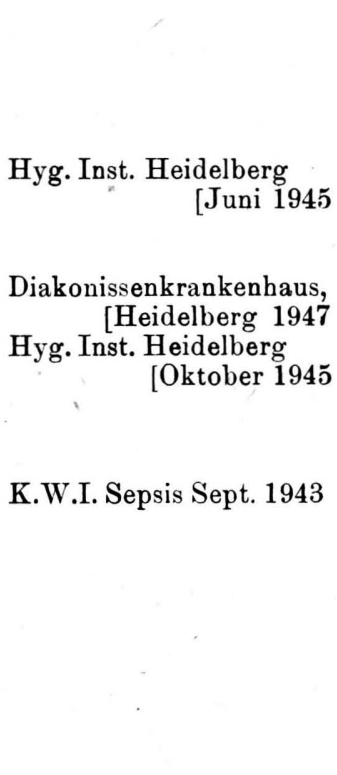 \\
\hline
\end{tabular}

Tab.3. Folinsäurebedürfnis von Staphylokokken (bei $37^{\circ}$ ). Zeichenerklärung: s. Tab. 2.

\section{Ergebnisse}

An Milchsäurebakterien, von denen bekannt ist, daß manche Stämme Folinsäure zum Wachstum brauchen, untersuchten wir 13 Stämme. Von diesen benötigten 6 Stämme unbedingt Folinsäure, 1 Stamm zeigte mit Folinsäure einen Zuwachseffekt, während die restlichen 6 Stämme ohne dieses Vitamin normales Wachstum zeigten (s.Tab.2).

Über das Folinsäurebedürfnis von Staphylo- kokken ist uns bisher nichts bekannt geworden. Wir untersuchten 25 Stämme und fanden, daß für 10 Stämme Folinsäure ein unbedingt notwendiger Wuchsstoff ist. 7 Stämme wurden nur im Wachstum gefördert, während die restlichen 8 Stämme in ihrem Wachstum von Folinsäure nicht beeinflußt wurden (s. Tab. 3). Von 2 Stämmen (Staph.aureus $\mathrm{KiAu}$ und Staph. aureus S) wurde das Folinsäurebedürfnis quantitativ, ebenso die Spezifität, untersucht, worüber weiter unten berichtet wird. 


\begin{tabular}{|l|l|c|c|}
\hline \multicolumn{1}{|c|}{ Bacterium } & Stamm & $\begin{array}{c}\text { Folinsäure- } \\
\text { bedürfnis }\end{array}$ & Herkunft der Stämme \\
\hline Streptococcus anhaemolyticus & $1646 \mathrm{Sc}$ & $\times$ & \\
Enterococcus & $1883 \mathrm{En}$ & + & \\
Micrococcus pyogenes & $\mathrm{MP}$ & 0 & \\
Micrococcus aureus & $\mathrm{Ma}$ & 0 & Süddeutsche Versuchs- und \\
Micrococcus roseus & $\mathrm{Mr}$ & 0 & Forschungsanstalt für \\
Sarcina lutea & $\mathrm{SL}$ & 0 & Milchwirtschaft, Weihenstephan \\
Sarcina flava & Sarc. & 0 & \\
\hline
\end{tabular}

Tab. 4. Folinsäurebedürfnis von verschiedenen Bakterien. Zeichenerklärung: s. Tab. 2.

In Tab. 4 ist das Folinsäurebedürfnis einiger weiterer Bakterien aufgeführt.

Folgende Bakterien wuchsen in folinsäurehaltigem und folinsäurefreiem Medium gleich stark:

Proteus-vulgaris-Stämme: X 19; O Pr; 1035 Pr; $1871 \mathrm{Pr} ; 1872 \mathrm{Pr} ; 1961 \mathrm{Pr} ; 2602$ Pr; 3609 Pr; 3747 Pr; 3799 Pr; 3880 Pr; 4332 Pr; 4406 Pr; 7466 Pr; 0964 Pr.

Bacterium-coli-commune-Stämme: OCo und Co1.

Bacterium-coli-imperfectum-Stämme: 3920 und 3920a. Weitere Coli-Stämme: 747 Co; 882 Co; $1883 \mathrm{Co} ; 1857$ Co.

Bacterium pyocyaneus: $0421 \mathrm{Py}$.

Bacterium-fluorescens-Stämme: Fl. I; Fl. II; SG $120 \mathrm{Fl}$.

Je ein Stamm von Bacterium dysenteriae: A; B; D; H; Schmitz; Strong; Shiga-Kruse; Flexner. Bacterium typhi; Bacterium paratyphi A und B; Bacterium paratyphi (enteritidis) Gaertner; Bacterium paratyphi (enteritidis) Breslau (Herkunft: Hyg. Inst. Heidelberg).

Die folgenden Stämme von Bact. diphtheriae: Bi 1240; Bi 1185; Bi 1125; Bi 1102 (Herkunft:
Hyg. Inst. Heidelberg) wurden durch Folinsäure bei der benutzten Konzentration etwas gehemmt.

Folinsäuretest mit Staphylococcus a ureus

Von der Beobachtung ausgehend, daß einige Stämme von Staph.aureus und Staph.albus unbedingt Folinsäure in dem verwendeten Nährmedium zum Wachstum benötigen (vgl.Tab.3), haben wir das Folinsäurebedürfnis und die Spezifität bei zwei Stämmen, nämlich Staph. aureus KiAu und Staph.aureus S näher untersucht.

In den Abb. $1 \mathrm{a}$ und $1 \mathrm{~b}$ sind Wachstumskurven für die beiden Stämme nach 15-, 23- und 48-stdg. Bebrütung aufgezeichnet. Eine weitere Bebrütung führte nicht mehr zu stärkerem Wachstum.

Zur quantitativen Bestimmung von Folinsäure ist natürlich nur der mittlere lineare Teil der Kurven verwendbar. In den Abb. $2 \mathrm{a}$ und $2 \mathrm{~b}$ ist dieser für Staph.aureus KiAu im Vergleich mit Sc.faecalis $\mathrm{R}$ dargestellt. Die Folinsäurekonzentration wurde in kleinen und linearen Intervallen gesteigert. Es zeigte sich, daß die Leerwerte bei dem
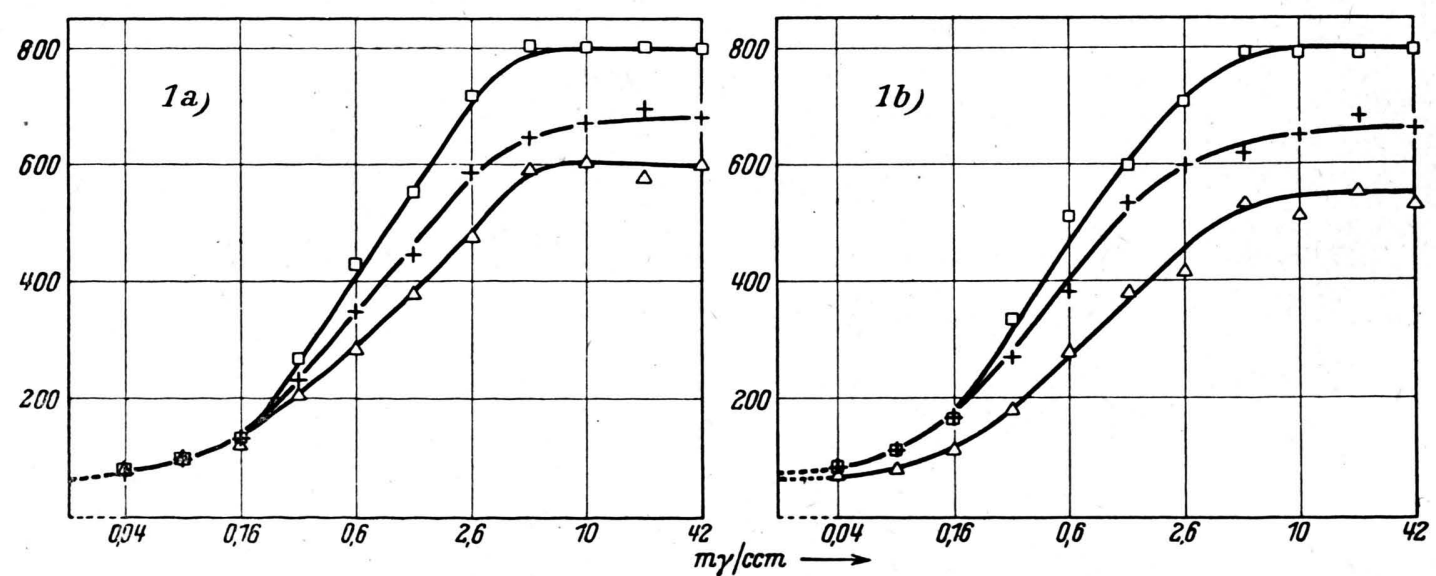

Abb. $1 \mathrm{a}$ und $1 \mathrm{~b}$. Wachstum von Staph.aureus in Abhängigkeit von der Folinsäurekonzentration nach 15 Stdn. $\triangle-\triangle, 23$ Stdn. +- und 48 Stdn. $\square-\square$ bei Stamm KiAu (Abb. 1a) und Stamm S (Abb. 1b). Abszissen: Konz. an Folinsäure in $\mathrm{m} \gamma / \mathrm{cm}^{3}$. Ordinaten: Extinktionswerte $\times 1000$ am Lange-Photometer. 
Staphylokokkus niedriger liegen als bei Sc. faecalis R. Die Nullwerte sind bei dem Staphylokokkus auffallend konstant, während die Streuungen im linearen Teil genau so gering sind wie bei $S c$. faecalis $\mathrm{R}$. Die Streuungen im optimalen Gebiet sind größer. Aus den Abb. 2 ergibt sich ferner, daß die Kurven für KiAu bereits nach 15 Stdn. praktisch linear sind und das Wachstum stark genug ist, während dies für Sc.faecalis $\mathrm{R}$ erst nach 20 Stdn. der Fall ist. Es ist für die Geschwindigkeit des Testes gleichgültig, ob mit einer Öse (Durchm. $2 \mathrm{~mm}$ ) oder mit $0,1 \mathrm{~cm}^{3}$ einer $24 \mathrm{Stdn}$.

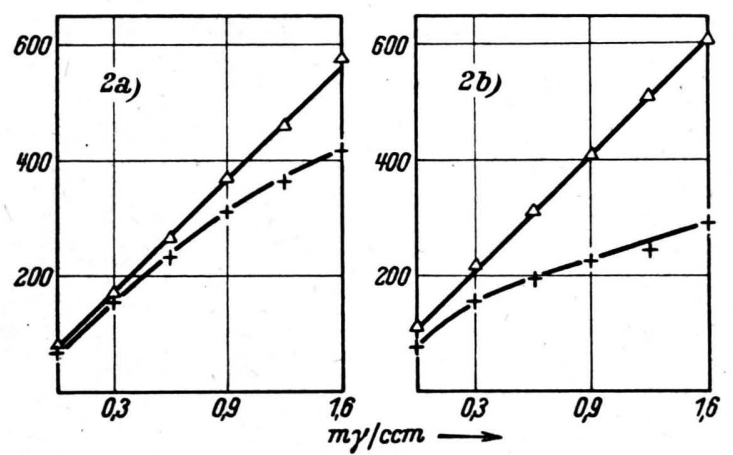

Abb. 2 a und 2 b. Linearer Teil der Wachstumskurven von Staph.aureus KiAu (Abb.2a) bzw. Sc.faecalis $\mathrm{R}$ (Abb. 2 b) nach 15 Stdn. +-+ und 24 Stdn. $\Delta-\Delta$. Mittelwerte aus je 7 Bestimmungen. Abszissen und Ordinaten wie in Abb. 1.

alten Kultur beimpft wird. Durch die Verwendung einer großen Impfmenge wird der Nullwert weder bei Staph.aureus, noch bei Sc.faecalis $\mathrm{R}$ erhöht. Das Alter der Impfkultur soll nicht größer als 24 Stdn. sein, da bei Verwendung älterer Impfkulturen die Wachstumsgeschwindigkeit sinkt.

Gegenüber dem bekannten Folinsäuretest mit Sc. faecalis $\mathrm{R}^{5}$ scheint uns bei dem neuen Test die leichte Zugänglichkeit geeigneter Staphylokokkenstämme der wesentliche Vorteil zu sein, neben den tieferen Nullwerten und der etwas größeren Wachstumsgeschwindigkeit.

Im Vergleich zu Sc.faecalis $\mathrm{R}$ war es erforder-

5 E. E.Snell u. W. H. P e ters on, J. Bacteriol. 39, 273 [1940]; T. D. L u c k e y, G. M. B r i g g s u. C. A. E l v e h j e m, J. biol. Chemistry 152, 157 [1944]; O. D. B ird, B. B r e Bler, R. A. B rown, C. J. C a m p be l l u. A. D. Emm et t, J. biol. Chemistry 159, 631 [1945]; H. K. M it chell u. E. E. S n ell, Univ. Texas Publ. Nr. 4137 [1941]; M. L a n d y u. D. M. D i c k e n, J. Lab. clin. Med. 27, 1086 [1942]; L. J. Teply u. C. A. Elvehjem, J. biol. Chemistry 157, 303 [1945]; E. C. Roberts u. E. E. S n el l, J. biol. Chemistry 163, 499 [1946].

\begin{tabular}{|l|c|c|c|}
\hline & $\begin{array}{c}\text { Staph. } \\
\text { aureus } \\
\text { KiAu }\end{array}$ & $\begin{array}{c}\text { Sc. faecalis } \\
\mathrm{R}\end{array}$ & $\begin{array}{c}\text { Sc. faecalis } \mathrm{R} \\
\text { nach der } \\
\text { Literatur }\end{array}$ \\
\hline Folinsäure & 1,0 & 0,8 & $0,3^{7}$ \\
Formylfolinsäure & 1,0 & 0,8 & $0,3^{8}$ \\
Pteroinsäure & 2,0 & 1,9 & $0,8^{7}$ \\
$\begin{array}{l}\text { Rhizopterin } \\
\text { Teropterin }\end{array}$ & 0,2 & 0,16 & $0,034^{9}$ \\
Thymin & 34 & $\mathbf{2 8}$ & $\mathbf{4 , 2}^{7}$ \\
\hline
\end{tabular}

Tab. 5. Konzentrationen verschiedener Stoffe in $\mathrm{m} \gamma / \mathrm{cm}^{3}$ für halboptimales Wachstum von Staph.aureus KiAu und Sc.faecalis R.

lich, auch bei Staph. aureus die mit der Folinsäure verwandten Verbindungen auf Wuchsstoffwirkung zu prüfen.

In den Abb. $3 \mathrm{a}$ und $3 \mathrm{~b}$ sind die Wirksamkeiten von Folinsäure, Formylfolinsäure, Pteroinsäure, Pteroyl-triglutaminsäure (= Teropterin ${ }^{6}$ ) und Thymin bei Staph. aureus KiAu (Abb. 3a) und Sc.faecalis R (Abb. 3 b) aufgetragen. Die Konzentrationen für halboptimales Wachstum in $\mathrm{m} \gamma / \mathrm{cm}^{3}$ sind für die beiden Bakterienstämme in Tab. $5 \mathrm{zu}$ finden. (Die Werte sind Mittel aus 4 bis 8 Einzelwerten, die ihrerseits um $\pm 10 \%$ streuen.)

4-Methyluracil zeigte bei Staph.aureus KiAu und Sc.faecalis $R$ in einer Konz. von $174 \gamma / \mathrm{cm}^{3}$ eine schwache, aber deutliche Wachstumswirkung.

Die in der letzten Spalte angegebenen, der Literatur entnommenen Wirksamkeiten wurden offenbar in Testen ermittelt, in denen säurehydrolysiertes Casein verwendet worden war. Da nach Roberts und $\mathrm{Snell^{3 }}$ die optimal wirksamen Konzentrationen der Folinsäure für Sc.faecalis $\mathrm{R}$ bei Anwendung von pankreatin-verdautem Casein 2,6-mal größer sind als mit säurehydrolysiertem Casein, ist die Übereinstimmung unserer Werte mit denen der Literatur befriedigend. Kleinere Unterschiede dürften darauf zurückzuführen sein, daß das Casein anderer Herkunft war und das Peptonpräparat weggelassen wurde. Die Konzentrationen für halboptimales Wachstum sind bei

6 Teropterin (Le d e r l e Laboratories), etwa 70\% Pteroyl-triglutaminsäure enthaltend.

7 C. W. W a l l e r et al., J. Amer. chem. Soc. 70, 19 [1948].

8 M. Gordon, J. M. R a vel, R. E. Eakin u. W. Shive, J. Amer. chem. Soc. 70, 878 [1948].

9 E. L. Rickers, L. Chalet u. J. Keresztesy, J. Amer. chem. Soc. 69, 2749 [1947].

${ }_{10} \mathrm{~K}$. K. K r u e g e r u. W. H. P e t e r s o n, J. biol. Chemistry 158, 145 [1945]. 

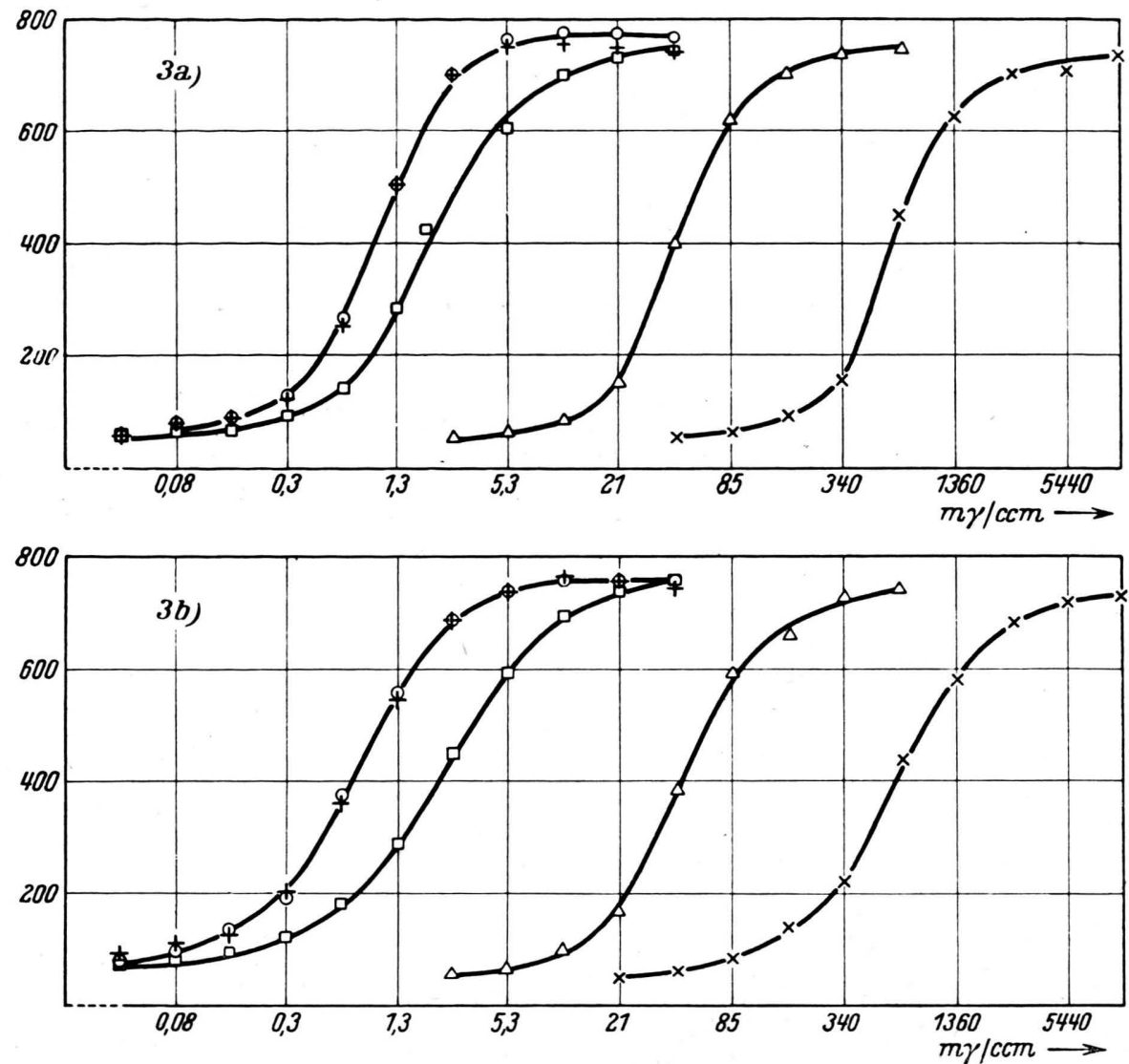

Abb. 3 a und 3b. Wachstum von Staph. aureus KiAu (Abb.3a) und Sc.faecalis R (Abb. 3b) in Anwesenheit steigender Mengen von $\circ-\circ$ Folinsäure, +- + Formylfolinsäure, $\square-\square$ Pteroinsäure, $\Delta-\Delta$ Pteroyltriglutaminsäure, $X-x$ Thymin. Abszissen: Konzentrationen in $\mathrm{m} \gamma / \mathrm{cm}^{3}$. Ordinaten: Extinktionswerte $\times 1000$ am Lang e-Photometer.

dem Staphylokokkenstamm KiAu für alle geprüften Verbindungen etwas höher als bei Sc.faecalis R.

Die Beobachtungen über den Antagonismus Folinsäure: 2 - Amino - 6 - oxy - pteridinaldehyd-(8) und die Wuchsstoffwirkung der Kombination dieses Aldehyds mit $p$-Aminobenzoesäure bzw. $p$ -
Aminobenzoyl-l-glutaminsäure bei Sc. faecalis R, worüber wir berichtet haben ${ }^{1}$, konnten wir auch bei den folinsäurebedürftigen Staphylokokken und verschiedenen Milchsäurebakterien machen. Näheres wird darüber später mitgeteilt werden.

Wir danken Frl. F. Uhl vom Hyg. Inst. Heidelberg für die Reinzüchtung verschiedener Bakterienstämme. 